\title{
Spatiotemporal Pattern of Motoneuron Activation in the Rostral Lumbar and the Sacral Segments during Locomotor-Like Activity in the Neonatal Mouse Spinal Cord
}

\author{
Agnès Bonnot, Patrick J. Whelan, George Z. Mentis, and Michael J. O’Donovan \\ Laboratory of Neural Control, Section on Developmental Neurobiology, National Institute of Neurological Disorders and \\ Stroke, National Institutes of Health, Bethesda, Maryland 20892
}

We used calcium imaging to visualize the spatiotemporal pattern of motoneuron activity during dorsal root-evoked locomotor-like bursting in the lumbosacral spinal cord of the neonatal mouse. Dorsal root stimuli elicited a tonic discharge in motoneurons on which alternating left-right rhythmic discharges were superimposed. Both the tonic and the rhythmic components could be recorded optically from populations of motoneurons labeled with calcium-green dextran. Optical and electrical recordings revealed that rhythmic signals from different parts of the lumbar (L1, L2) and sacral (S1-S3) segments rose, peaked, and decayed in a rostrocaudal sequence. This pattern gave rise to a rostrocaudal "wave" in the activation of motoneurons during each cycle of locomotor-like activity. A similar rostrocaudal delay was observed during episodes of alternation that occurred in the absence of stimulation, suggesting that this delay was not caused by the train of dorsal root stimuli. It is hypothesized that this behavior may simplify the appropriate sequencing of motoneurons during locomotion.

Key words: calcium imaging; motoneuron; locomotion; spinal cord; rhythmogenesis; mouse
During locomotion, the motoneuron pools innervating the limbs, the axial musculature, and the tail are activated in a complex spatiotemporal pattern. Little is known about the mechanisms that ensure the proper coordination between motoneurons during this behavior. In several species, including the cat, rat, turtle, and chick embryo, it appears that the most rostral segments of the lumbar cord are the most excitable (for review, see Kiehn and Kjaerulff, 1998), and there is some evidence that they may drive the more caudal segments (Cazalets and Bertrand, 2000). However, other studies have established that rhythmogenicity is distributed along the lumbosacral cord and can be expressed in both the isolated lumbar and sacral cords (Whelan et al., 2000; Delvolvé et al., 2001).

During forward swimming, the motoneurons of fish, frogs, tadpoles, and urodeles are activated in a rostrocaudal sequence (Kahn et al., 1982; Wallen and Williams, 1984; Delvolvé et al., 1997), although such sequences can be reversed under some conditions (Delvolvé et al., 1999). In principle, a rostrocaudal pattern of activation would greatly simplify the problem of recruiting hindlimb motoneurons in the appropriate sequence during locomotion by exploiting their rostrocaudal position along the axis of the lumbar cord. However, it is not known whether this pattern, or a variant of it, operates to coordinate the lumbar and sacral motoneurons during locomotion in mammals. Obviously, the activation of motoneurons during locomotion will be more complex than during swimming, because the limb-moving seg-

\footnotetext{
Received July 27, 2001; revised Sept. 20, 2001; accepted Nov. 6, 2001.

We thank Arthur Prochazka and Peter Wenner for comments on this paper and Bill Marks for statistical advice.

Correspondence should be addressed to Agnes Bonnot, Laboratory of Neural Control, Section on Developmental Neurobiology, National Institute of Neurological Disorders and Stroke, National Institutes of Health, Bethesda, MD 20892. E-mail: bonnota@ninds.nih.gov.

Dr. Whelan's present address: Department of Physiology and Biophysics, University of Calgary, Calgary, Alberta, Canada T2N 4N1.

Copyright (C) 2002 Society for Neuroscience $0270-6474 / 02 / 220001-06 \$ 15.00 / 0$
}

ments are composed of flexor and extensor motoneurons that are activated out of phase. Recently, Yakovenko et al. (2002) performed a modeling study in which they combined published EMG data from cat hindlimb motoneurons during treadmill locomotion with anatomical data about the position of motoneurons along the spinal cord (Vanderhorst and Holstege, 1997) to generate a model of the spatiotemporal pattern of motoneuron activity in the cat spinal cord during walking. This study suggested that motoneuron activity was oscillating rostrocaudally during the flexor and extensor phases of the locomotor cycle.

In the present work, we have used calcium imaging to visualize the spatiotemporal spread of activity in the rostral lumbar and sacral segments during rhythmic activity induced by dorsal root stimulation in the neonatal mouse spinal cord. This activity is locomotor-like in both the rat (Marchetti et al., 2001) and the mouse (Bonnot et al., 2002), because the lumbar ventral root discharge alternates between the left and right sides and between flexor and extensor pools. We focused on the activity of the rostral lumbar and the sacral segments because their constituent motoneurons fire in phase (Whelan et al., 2000). Some of this work has been published previously in abstract form (Bonnot et al., 1998, 1999, 2001).

This article is published in The Journal of Neuroscience, Rapid Communications Section, which publishes brief, peerreviewed papers online, not in print. Rapid Communications are posted online approximately one month earlier than they would appear if printed. They are listed in the Table of Contents of the next open issue of JNeurosci. Cite this article as: JNeurosci, 2002, 22:RC203 (1-6). The publication date is the date of posting online at www.jneurosci.org.

http://www.jneurosci.org/cgi/content/full/6031 


\section{MATERIALS AND METHODS}

Experiments were performed on 19 Swiss Webster mice (Taconic Laboratory, Germantown, NY) that were $1-4 \mathrm{~d}$ of age [postnatal day 0 (P0)-P3]. After induction of anesthesia with methoxyflurane, the animals were decapitated and eviscerated. The remaining tissue was placed in a dissecting chamber containing artificial CSF (ACSF) (concentration in $\mathrm{mm}$ ): $128 \mathrm{NaCl}, 4 \mathrm{KCl}, 1.5 \mathrm{CaCl}_{2}, 1 \mathrm{MgSO}_{4}, 0.5 \mathrm{NaH}_{2} \mathrm{PO}_{4}, 21 \mathrm{NaHCO}_{3}$, and $30 \mathrm{D}$-glucose, bubbled with $95 \% \mathrm{O}_{2}$ and $5 \% \mathrm{CO}_{2}$. A ventral laminectomy exposed the cord, which was then transected at T5-T7 and removed from the vertebral column together with the cauda equina. Full details have been described previously (Whelan et al., 2000).

Dye loading. In 14 experiments, motoneurons spanning two to three segments (T13-L2 or S1-S3) were retrogradely loaded with calciumgreen 1 (dextran-conjugated, molecular weight of 10,000; Molecular Probes, Eugene, OR) using established techniques (O'Donovan et al., 1993). For this purpose, several adjacent ventral roots were drawn into a suction electrode containing $\sim 6 \mu \mathrm{l}$ solution of the dye $(10 \mathrm{~mm})$ dissolved in distilled $\mathrm{H}_{2} \mathrm{O}$ with $0.2 \%$ Triton $\mathrm{X}-100$. The cord was left at $17^{\circ} \mathrm{C}$ for $8-10 \mathrm{hr}$ to allow labeling of cell bodies.

Optical imaging. The cord was placed in a chamber on an inverted epifluorescence microscope (Eclipse TE300; Nikon, Tokyo, Japan) and continuously perfused with ACSF. It was illuminated with a $75 \mathrm{~W}$ xenon light source (excitation filter, $470-490 \mathrm{~nm}$; emission filter, $520-560 \mathrm{~nm}$ ), and labeled motoneurons were visualized through the ventral white matter. Activity-dependent changes in fluorescence were detected using an intensified CCD camera (Stanford Photonics, Salt Lake City, UT) and stored on videotape (SVO 9500MD; Sony, Tokyo, Japan). Changes in fluorescence were monitored on-line using a PC-based frame grabber (Matrox Image LC; Matrox Graphics, Dorval, Quebec, Canada) and image-processing software (Metamorph; Universal Imaging, West Chester, PA).

Electrical recordings and activation of the locomotor networks. Motoneuron electrical activity was recorded together with optical recordings (eight experiments) or alone (five experiments) with suction electrodes into which individual ventral roots were drawn. The electrical signals were filtered (DC, $3 \mathrm{kHz}$ ), digitized (NeuroData, New York, NY), and recorded on videotape. Locomotor-like activity was evoked by electrical stimulation of the cauda equina or individual coccygeal dorsal roots (trains, 4-8 Hz; stimulus duration, $500 \mu \mathrm{sec}$; stimulus intensity, 20-200 $\mu \mathrm{A}$; train duration, $10 \mathrm{sec})$ or with a brief suction pulse $(1-2 \mathrm{sec})$ applied to the dorsal root. Spinal motoneurons were activated antidromically by stimulation of the ventral roots used to load motoneurons (stimulus duration, $500 \mu \mathrm{sec}$; trains, $5-50 \mathrm{~Hz}$; train duration, $10 \mathrm{sec}$ ).

Data analyses. Stacks of images were collected off-line during periods of evoked activity, spontaneous activity, or antidromic stimulation (30 frames/sec). Synchronization of the electrical and optical signals was achieved during 8 of 11 experiments by gating the camera off and recording the gating pulse together with the electrical recordings. Electrical activity was filtered $(100-500 \mathrm{~Hz})$, digitized $(1 \mathrm{kHz})$, and integrated at $30 \mathrm{~Hz}$ for comparison with time series of the fluorescence measurements.

Both optical and electrical data were analyzed using crosscorrelograms to examine the left-right phasing and the rostrocaudal spread of activity during rhythmic episodes (Fig. 1). The data were smoothed with a five point moving average, demeaned and detrended before computing cross-correlograms between the various regions or roots using commercially available software (Statistica, Tulsa, OK). Analyses were performed on records that exhibited regular rhythmic discharges (observation of the correlograms over two to three cycles; 100 lags; lag duration of $33 \mathrm{msec}$ ) alternating between the left and right sides of the cord (phase value of $0.5 \pm 0.01$, measured optically or electrically at the same rostrocaudal level).

Regions of interest were drawn over motoneuronal populations (four to five regions per segment, $\sim 140 \mu \mathrm{m}$ apart). We generated crosscorrelograms between the optical signal of the most caudal region of interest (reference signal) and the optical signals at successively more rostral positions along the cord. When performed this way, a positive delay in the peak of the cross-correlogram indicates that the caudal signal was delayed with respect to the rostral signal. Distances between regions of interest (range of 100-1300 $\mu \mathrm{m}$ ) were measured from the center of the most caudal region to the center of every other region (see Fig. $3 B$ ). The delays between the regions were obtained from the $\mathrm{X}$ coordinates of the central peaks of the resulting cross-correlograms (peaks estimated using a polynomial fit). To determine whether the delay increased significantly with the distance from the most caudal region, a linear regression
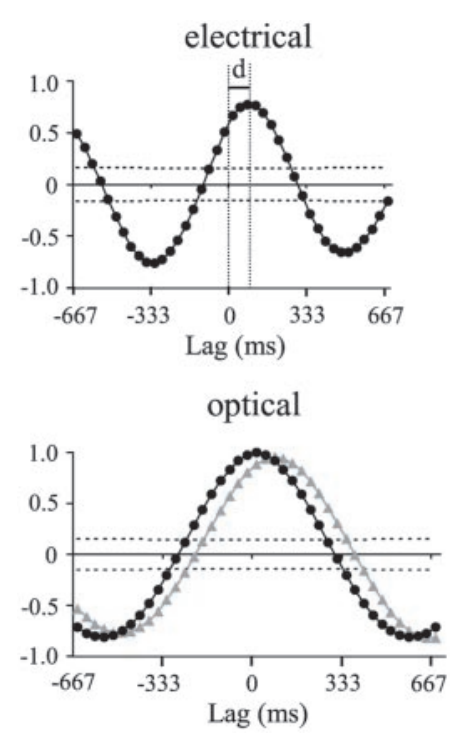

Figure 1. Measurement of the rostrocaudal spread of electrical and optical activity. Top panel, Cross-correlogram generated from a spontaneous episode (seven cycles) of ventral root discharge recorded from the ipsilateral L1 and L2 ventral roots. The distance between 0 and the peak of the cross-correlogram (L2/L1) corresponds to the delay $(d)$ between L1 and L2. Bottom panel, Correlograms generated from the optical activity illustrated in Figure $3 A$. The black trace (circles) shows the autocorrelogram of the optical signal recorded from the most caudal region of the L2 segment (Fig. 3B, blue arrow). The gray trace (triangles) is the crosscorrelogram between the optical activity of the region that was autocorrelated and the most rostral region of the L1 segment (Fig. 3B, red arrow). The distance between the two peaks corresponds to the delay between those two regions. In both graphs, the horizontal dotted lines indicate \pm 2 SE.

analysis was performed between delays and distances (correlation coefficient), and a $t$ test was used to establish the probability that the variables were not significantly correlated. The same approach was used to examine the correlation between the propagation velocity and the cycle period.

Confocal microscopy. Spinal cords in which lumbar (two of four cords) or sacral (two of four cords) motoneurons had been labeled previously were fixed in 1-ethyl-3-(dimethylaminopropyl) carbodiimide hydrochloride (EDC; Sigma-Aldrich, St. Louis, MO). EDC was prepared fresh at a concentration of $40 \mathrm{mg} / \mathrm{ml}$ in PBS (Tymianski et al., 1997). The spinal cords were refrigerated and sectioned the following day. The fixed cords were washed in PBS for 5-10 min and subsequently embedded in 5\% agar in PBS. The cords were cut horizontally $(70 \mu \mathrm{m})$ or transversely $(50 \mu \mathrm{m})$ using a Vibratome. Sections were collected on slides, mounted with an antifading solution (7:3 PBS/glycerol), and coverslipped. Sections were viewed on a Zeiss (Thornwood, NY) LSM410 confocal microscope (excitation, $488 \mathrm{~nm}$; emission, 515-540 nm) immediately after sectioning. Images presented in this study are two-dimensional projections from a $\mathrm{z}$-series collected at $1-5 \mu \mathrm{m}$ intervals for $40-80 \mu \mathrm{m}$.

\section{RESULTS}

\section{Retrograde loading of motoneurons and optical signals during antidromic stimulation}

Figure $2 A-C$ shows confocal images of lumbar and sacral motoneurons labeled with calcium-green dextran applied to the cut ventral roots. The labeling pattern and distribution of motoneurons in the lumbar cord are shown in horizontal (L2-L3; Fig. 2A) and transverse (L2; Fig. $2 B$ ) sections. In both the anterior lumbar (L1/L2) and the S1 sacral segment, the motoneurons extended as a flattened pool with a dorsoventral thickness of $\sim 100-150 \mu \mathrm{m}$.

We first established that discharge could be detected optically from the labeled motoneurons. This was accomplished in 10 experiments by antidromic stimulation of the ventral root of a 

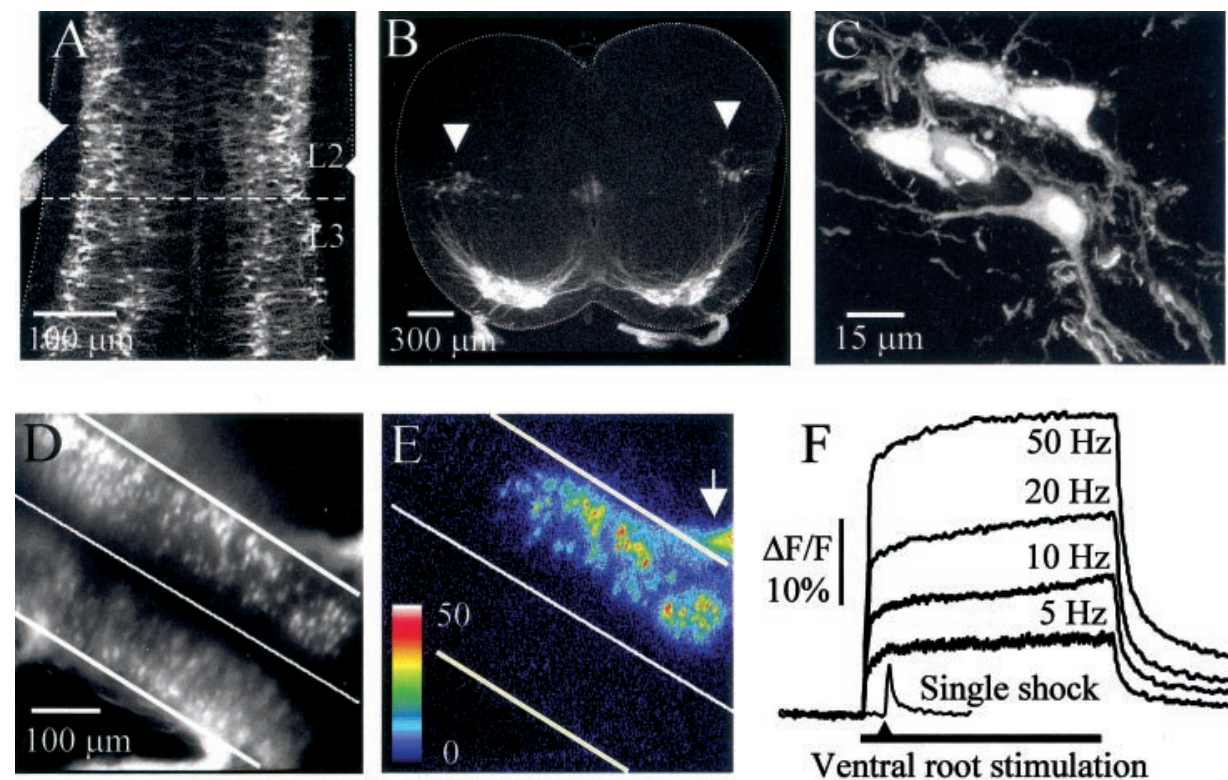

Figure 2. $A-C$, Confocal photomicrographs of retrogradely labeled motoneurons. $A$ Horizontal section (L2-L3). The dashed line indicates the limit between L2 and L3. B, Transverse section (L2) showing motoneurons and presumed preganglionic neurons (arrowheads). C, High-power view showing labeled sacral (S1) motoneurons in a transverse section. $D$, Fluorescence microgram of the S2 and S3 segments viewed through the ventral white matter. $E$, Difference image obtained by subtracting the control fluorescence from an average generated during stimulation of the S2 ventral root (arrow) at $20 \mathrm{~Hz} . F$, Fluorescence transients generated from motoneurons (same experiment as in $D$ and $E$ ) in response to antidromic stimulation (single shock at $50 \mu \mathrm{A}$ and $10 \mathrm{sec}$ trains at different frequencies). The dotted $(A, B)$ or solid $(D, E)$ lines outline the spinal cord. labeled segment and recording of the evoked optical signals (Fig. $2 D-F)$. When the ventral root was stimulated, the activity of the entire segment of labeled motoneurons could be visualized (Fig. $2 E$ ). We examined the frequency dependence of the antidromic signal between 1 and $20 \mathrm{~Hz}$ in four experiments and up to $50 \mathrm{~Hz}$ in two of these experiments (Fig. $2 F$ ). After a single shock, the peak fractional change in fluorescence averaged $8.7 \pm 4.2 \%$ (mean \pm SD, range of 4.3-14.4\%). At a stimulus frequency of 20 $\mathrm{Hz}$, the corresponding values were $30.9 \pm 5.4 \%$ (range of $26.8-$ $36.1 \%$ ). These results indicate that calcium dyes can be used to resolve motoneuron discharge in the neonatal mouse spinal cord at the resolution of single action potentials (Fig. $2 F$, Single shock). The cellular variation in the intensity of the optical signal (Fig. $2 E$ ) arises in part because of differences in the amount of dye loaded into individual motoneurons. When this is corrected, by normalizing the fluorescence change, this variation is much less. It is also possible that calcium influx generated by action potentials varies between different types of motoneurons.

\section{Motoneuron electrical and optical signals during locomotor-like activity induced by dorsal root stimulation}

A train of dorsal root stimuli induced rhythmic discharges that alternated between the left and right sides of the cord and persisted for the duration of the stimulus (Fig. 3A) (Whelan et al., 2000). During the induced bursting, large optical signals (peak fractional change in fluorescence $\Delta F / F$ measured from baseline, $41.0 \pm 8.9 \%$; 38 episodes, 11 animals) were recorded from the labeled motoneurons (Fig. $3 A$ ). The optical signals comprised a tonic component $(\Delta F / F, 21.2 \pm 5.5 \%)$ and a rhythmic component $(\Delta F / F$ measured from the tonic component, $19.8 \pm 7.3 \%)$. As illustrated in Figure 3, each cycle of rhythmic optical activity coincided with the ipsilateral ventral root discharge and alternated with the optical and electrical activity recorded contralaterally. Because action potentials appear to be the primary determinant of the motoneuronal calcium signals (O'Donovan et al., 1993; Lev-Tov and O'Donovan, 1995), we presume that the tonic optical signal arises from the temporal summation of the calcium transients accompanying individual bursts of motoneuron discharge.
To assess the spread of activity along the cord, we monitored the optical signals at multiple locations along the rostrocaudal axis, as illustrated in Figure $3 B$. Typically, we monitored four to five adjacent regions within each segment (the arrows in Fig. $3 B$ show the center of every other region). We performed this analysis across single and multiple segments in the thoraco-lumbar (five experiments) and sacral (seven experiments) cord in 61 sequences of rhythmic activity obtained from 38 episodes of alternating left-right activity (11 animals). At the beginning of the episode, when the dorsal root stimuli induced the first rise in fluorescence, we found that activity often evolved in a caudorostral direction (data not shown). However, the pattern changed once rhythmic alternating bursting became well established. Under these conditions, the optical activity rose throughout the first part of each cycle to peak at approximately mid-cycle and then decayed during the remainder of the cycle (Fig. $3 C$ ). During each burst there was a progressive delay in the timing of the optical transients obtained from successively more caudal parts of the segments. This resulted in a rostrocaudal propagation of activity at a velocity of $15.0 \pm 8.1 \mu \mathrm{m} / \mathrm{msec}$.

The propagating component of the optical activity could be seen most strikingly in a video that displays only the rhythmic component of the optical signal (see video). Frames of such a video are illustrated in Fig. $3 D$, which shows the optical signals during a single cycle of rhythmic activity. In this example, the activity on the right side of the cord started at the asterisk and propagated over two segments within 150-180 msec. This was followed by a similar wave in the activity on the left side (Fig. 3D, bottom panels). We observed a rostrocaudal propagation of optical activity in 24 of 24 rhythmic sequences recorded from the thoraco-lumbar cord (16 alternating episodes, five experiments) and in 33 of 37 rhythmic sequences obtained from sacral segments (22 alternating episodes, seven experiments). The delay between the optical activity of different regions increased significantly with distance $(r=0.68 ; p<0.005)$. Only 83 of $372(\sim 22 \%)$ values of the delay computed between different regions revealed an absence of propagating activity or a short caudorostral sequence of activation. 

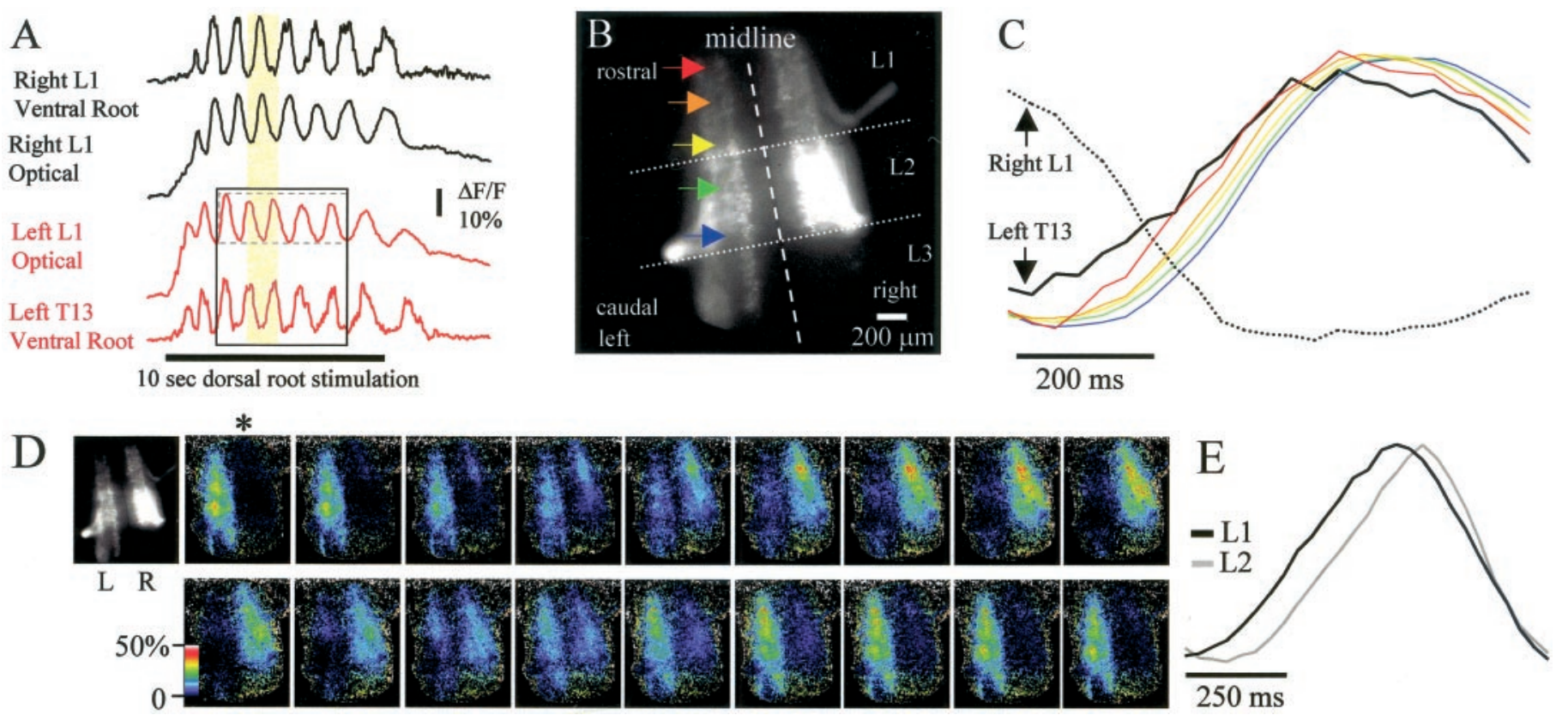

Figure 3. A, Rostrocaudal propagation of motoneuron optical signals recorded during locomotor-like activity induced by stimulation of a dorsal root $(30 \mu \mathrm{A}, 4 \mathrm{~Hz})$. The time course of the optical signals measured from the labeled motoneurons on both sides of the L1 segment (shown in $B$ ) was compared with that of the electrical activity recorded from the right L1 and left T13 ventral roots. Note that the electrical and optical activity alternates between the left and right sides (highlighted). B, Videomicrograph of the labeled motoneurons (L1-L3) viewed through the ventral white matter. The colored arrows indicate the center of the regions of interest. The midline (dashed line) and the limits between L1, L2, and L3 segments (dotted lines) are indicated. $C$, Average of five successive fluorescence transients (indicated by the dotted box in $A$ ) obtained from the regions marked in $B$ (same color code) compared with the averaged discharge recorded from the right $\mathrm{L} 1$ and the left T13 ventral roots (cycles indicated by the boxed area in $A$ ). $D$, Rostrocaudal spread of activity during a single cycle of rhythmic activity (indicated by gray bar in $A$ ). The first panel is the same as $B$, and subsequent panels are difference images normalized to the quiescent baseline. The trough in the normalized activity of each side was subtracted from the resulting images to demonstrate the propagation of activity during the rhythmic component of the signal. Every other video frame is illustrated. In this example, the rostrocaudal wave starts on the right side $(R)$ marked by the asterisk and progresses rostrocaudally. The first row shows the propagation on the right side and the second row shows the propagation on the left side (see accompanying video). E, Average of eight successive bursts of electrical activity in L1 and $\mathrm{L} 2$ ventral roots obtained during a train of dorsal root stimuli $(25 \mu \mathrm{A}, 4 \mathrm{~Hz})$.

We also found a significant negative correlation $(r=0.21 ; p<$ $0.005)$ between the velocity of rostrocaudal propagation and the cycle period. The cycle period was $\sim 15 \%$ shorter in the lumbar segments $(1.2 \pm 0.3 \mathrm{sec})$ compared to the sacral segments $(1.3 \pm$ $0.5 \mathrm{sec})$, and propagation was correspondingly faster in the lumbar $(17.5 \pm 9.1 \mu \mathrm{m} / \mathrm{msec})$ compared to the sacral $(12.5 \pm 6.1$ $\mu \mathrm{m} / \mathrm{msec}$ ) segments. Combining the average propagation velocity with the average length of a segment $(\sim 700 \mu \mathrm{m})$, the average delay along a single segment was $\sim 50 \mathrm{msec}$.

We found an average delay of $28 \pm 10 \mathrm{msec}$ (30 episodes, five animals) between the electrical activity of adjacent ventral roots (L1/L2, L2/L3, S1/S2, and S2/S3) during episodes of locomotorlike activity (Fig. $3 E$ ). As illustrated in Figures $3 C, E$ and $4 C, E$, the rising phase of both the optical and the integrated electrical activity was successively delayed along the cord, consistent with the idea that the onset of each cycle of activity was also delayed rostrocaudally.

\section{Spatiotemporal pattern of spontaneous activity}

It is possible that the rostrocaudal pattern of activation we observed was caused by the train of dorsal root stimuli rather than the evoked bursting. To test this possibility, we examined bursting (in the absence of stimulation) in several episodes that either occurred spontaneously (one optical and three electrical experiments) (Fig. 4) or were initiated by a brief mechanical stimulus
(1-2 sec suction pulse) applied to the dorsal root (one optical and two electrical experiments).

At the onset of a spontaneous episode recorded optically, the fluorescence change propagated caudorostrally (as it does at the onset of evoked alternation). However, once the alternation was established, a rostrocaudal propagation was detected with an average delay of $58 \mathrm{msec}$ along the segment (four bursts from three episodes of one or two alternating cycles). In the electrical experiments (Fig. $4 D-E$ ), we recorded a delay of $\sim 33 \mathrm{msec}$ (range of 15-65 msec, 14 episodes of 2-10 cycles) between the spontaneous discharges of ipsilateral adjacent ventral roots (L1/ $\mathrm{L} 2, \mathrm{~L} 2 / \mathrm{L} 3, \mathrm{~S} 1 / \mathrm{S} 2$, and S2/S3).

In the three experiments in which alternating activity was triggered by a brief mechanical stimulus (eight episodes of 5-13 cycles), we found delays similar to those measured during a train of dorsal root stimuli. In one optical experiment, the rostrocaudal propagation velocity across $\mathrm{L} 1 / \mathrm{L} 2$ was $16.9 \pm 3.5 \mu \mathrm{m} / \mathrm{msec}$ during a train of dorsal root stimuli (four episodes) and was $13.3 \pm 4.7$ $\mu \mathrm{m} / \mathrm{msec}$ (three episodes) after the mechanical stimulus. Similarly, in two experiments using ventral root recordings only, we found a delay of $25.0 \pm 9.4 \mathrm{msec}$ (six episodes) between the activity of adjacent sacral roots (S1/S2 or S2/S3) during the stimulus train and a delay of $27.8 \pm 7.3 \mathrm{msec}$ in five episodes initiated by the brief mechanical stimulus.

Collectively, these findings are consistent with the idea that 


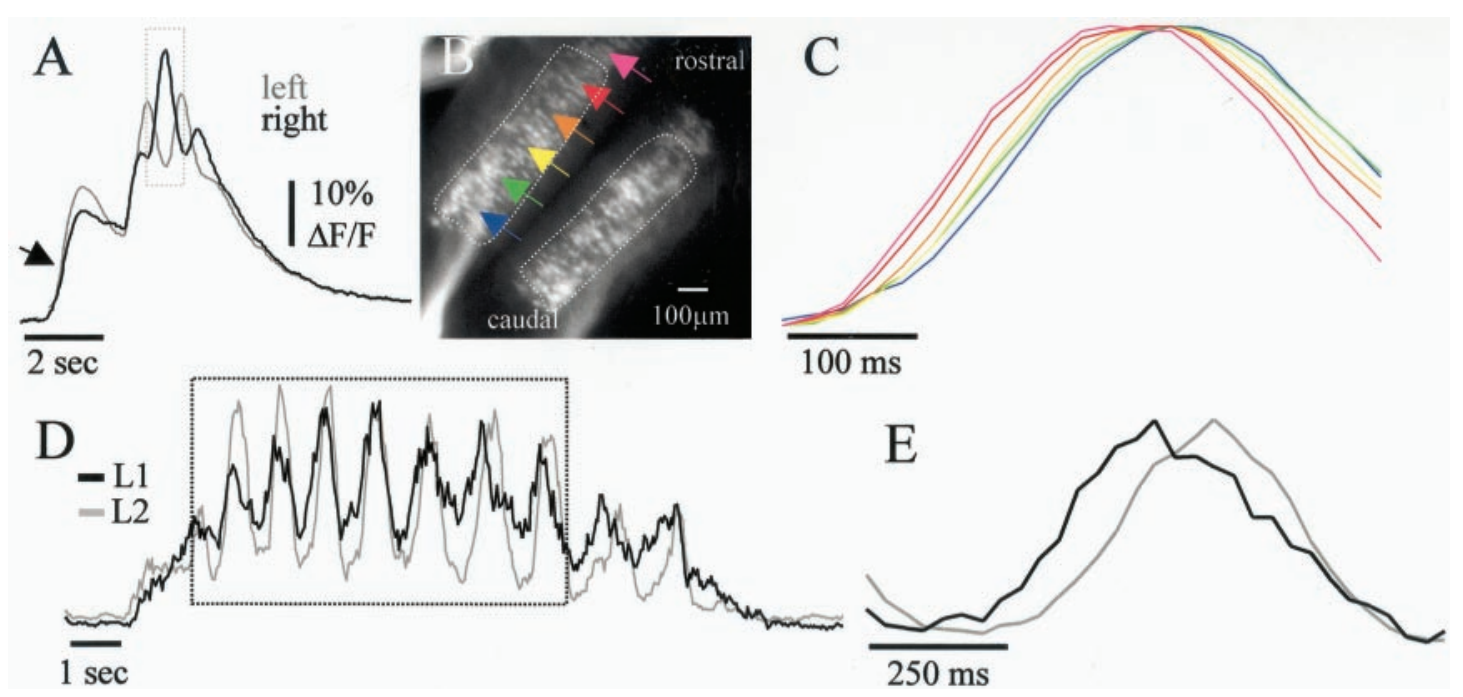

Figure 4. Electrical and optical recordings during spontaneous episodes of left-right alternation. $A$, Optical recording from labeled sacral motoneurons (averaged from the dotted regions in $B$ ) during a brief episode of spontaneous alternation. Note the synchronous activation of the left and right sides (arrow) before alternation develops. B, Videomicrograph of the labeled motoneurons (S2) viewed through the ventral white matter. The colored arrows indicate the center of the regions of interest. $C$, Normalized fluorescence transients measured from the regions of interest shown in $B$ (same color code) during the cycle on the left side of the cord (indicated by the dotted box in $A$ ). $D$, Integrated electrical activity of L1 and L2 ipsilateral ventral roots during a spontaneous episode of left-right alternation (same experiment as in Fig. 3E). E, Average of seven successive bursts (indicated by the dotted box in $D$ ).

rostrocaudal propagation is a characteristic of the bursting rather than the stimulus train.

\section{DISCUSSION}

We have identified a rostrocaudal "wave" in the activation of motoneurons of the first two lumbar and the first three sacral segments. Because the optical signals are likely to reflect spiking rather than subthreshold synaptic activity (O'Donovan et al., 1993; Lev-Tov and O'Donovan, 1995), our findings suggest that the propagating optical activity is generated by a corresponding propagation in the discharge of motoneuron populations. The optical findings were supported by the observation of a rostrocaudal delay in the discharge of adjacent ventral roots. However, because the electrical recordings sum the activity of motoneurons within the segment, it would not have been possible to establish the existence of a systematic rostrocaudal propagation within a segment based solely on such recordings.

We focused on the thoraco-lumbar segments (T13, L1/L2) and on the sacral segments for several reasons. First, previous work has shown that the ventral root discharges of these segments are coactive with flexor motoneurons during each cycle (Whelan et al., 2000). In contrast, the bursting of the L3-L5 ventral roots is more complex, comprising double bursts that probably correspond to activity of the flexor and extensor motoneurons known to be present in these segments (McHanwell and Biscoe, 1981). In addition, all ipsilateral motoneurons (flexors, extensors, and abductors) in the sacral segments have been shown to be coactive during dorsal root-evoked bursting in the neonatal rat (Delvolvé et al., 2001). We hypothesized that a propagating component of the optical signal would be easier to resolve within a single functional class of motoneurons. In future experiments, it will be useful to establish whether a propagating component of the optical activity can be detected within the flexor and extensor pools of the L3-L6 segments.

An important question that arises from these results is whether the rostrocaudal propagation of activity is attributable to the dorsal root train used to evoke the bursting. This might occur for example if a stimulus train applied to the cauda equina established a rostrocaudal gradient of excitability along the cord. Although we cannot exclude this possibility, we consider it unlikely for the following reasons. First, the stimuli during the dorsal root train $(4-8 \mathrm{~Hz})$ were never coupled with the rhythmic activity $(\sim 1.3 \mathrm{~Hz})$ and individually did not result in a rostrocaudal propagation. Second, and most compellingly, we found a rostrocaudal propagation of activity similar to that recorded during the dorsal root train, during spontaneous episodes, and during episodes triggered by a brief mechanical stimulus applied to the dorsal roots.

It is not clear whether the rostrocaudal pattern of activation we have found bears any relation to the patterns of motoneuron activity observed by Yakovenko et al. (2002) in their modeling studies of the cat step cycle. They found a rostrocaudal-caudorostral oscillation of activity during each step cycle that could be accounted for in part by the rostrocaudal separation of flexor and extensor motoneurons along the cord (flexor rostral-extensor caudal; Vanderhorst and Holstege, 1997). It is also possible that the pattern we have observed is entirely developmental, perhaps depending on the electrical coupling between neonatal motoneurons (Tresch and Kiehn, 2000). In future experiments, it will be very interesting to establish whether other modes of activation (e.g., caudorostral) can occur, as has been reported under certain conditions in other species (Delvolvé et al., 1999), and to determine the spatiotemporal pattern of activity during the much slower rhythms induced by drugs.

These findings raise the possibility that the lumbosacral motoneurons of mammals may be activated in part by mechanisms similar to those documented for the rostrocaudally organized swimming movements of simpler vertebrates. Such a pattern would greatly simplify the problem of ensuring proper coordination between the different pools of motoneurons by exploiting their position along the rostrocaudal axis of the cord. 


\section{REFERENCES}

Bonnot A, Tabak J, Cohen U, Wenner P, O'Donovan M (1998) Calcium imaging of rhythmic network activity in the mouse spinal cord. Soc Neurosci Abstr 24:1668.

Bonnot A, Whelan P, O'Donovan M (1999) Calcium imaging of spinal network behavior during locomotor-like activity in the neonatal mouse. Soc Neurosci Abstr 25:1904.

Bonnot AC, Whelan PJ, Mentis GZ, O'Donovan MJ (2001) Spatiotemporal pattern of motoneuron activation in the lumbosacral spinal cord of the neonatal mouse in vitro. Soc Neurosci Abstr 27:297.6.

Bonnot A, Whelan PJ, Mentis GZ, O’Donovan MJ (2002) Locomotorlike activity generated by the neonatal mouse spinal cord. Brain Res Rev, in press.

Cazalets JR, Bertrand S (2000) Coupling between lumbar and sacral motor networks in the neonatal rat spinal cord. Eur J Neurosci 12:2993-3002.

Delvolvé I, Bem T, Cabelguen JM (1997) Epaxial and limb muscle activity during swimming and terrestrial stepping in the adult newt, Pleurodeles waltl. J Neurophysiol 78:638-650.

Delvolvé I, Branchereau P, Dubuc R, Cabelguen JM (1999) Fictive rhythmic motor patterns induced by NMDA in an in vitro brain stem-spinal cord preparation from an adult urodele. J Neurophysiol 82:1074-1077.

Delvolvé I, Gabbay H, Lev-Tov A (2001) The motor output and behavior produced by rhythmogenic sacrocaudal networks in spinal cords of neonatal rats. J Neurophysiol 85:2100-2110.

Kahn JA, Roberts A, Kashin SM (1982) The neuromuscular basis of swimming movements in embryos of the amphibian Xenopus laevis. J Exp Biol 99:175-184.

Kiehn O, Kjaerulff O (1998) Distribution of central pattern generators for rhythmic motor output in the spinal cord of limbed vertebrates. In: Neuronal mechanisms for generating locomotor activity (Kiehn $\mathrm{O}$,
Harris Warrick RM, Jordan LM, Hultborn H, Kudo N, eds), 110-129. New York: New York Academy of Sciences.

Lev-Tov A, O'Donovan MJ (1995) Calcium imaging of motoneuron activity in the en-bloc spinal cord preparation of the neonatal rat. J Neurophysiol 74:1324-1334.

Marchetti C, Beato M, Nistri A (2001) Alternating rhythmic activity induced by dorsal root stimulation in the neonatal rat spinal cord in vitro. J Physiol (Lond) 530:105-112.

McHanwell S, Biscoe TJ (1981) The localization of motoneurons supplying the hindlimb muscles of the mouse. Philos Trans R Soc Lond B Biol Sci 293:477-508.

O'Donovan MJ, Ho S, Sholomenko G, Yee W (1993) Real-time imaging of neurons retrogradely and anterogradely labelled with calciumsensitive dyes. J Neurosci Methods 46:91-106.

Tresch MC, Kiehn O (2000) Motor coordination without action potentials in the mammalian spinal cord. Nat Neurosci 3:593-599.

Tymianski M, Bernstein GM, Abdel-Hamid KM, Sattler R, Velumian A, Carlen PL, Razavi H, Jones OT (1997) A novel use for a carbodiimide compound for the fixation of fluorescent and non-fluorescent calcium indicators in situ following physiological experiments. Cell Calcium 21:175-183.

Vanderhorst VG, Holstege G (1997) Organization of lumbosacral motoneuronal cell groups innervating hindlimb, pelvic floor, and axial muscles in the cat. J Comp Neurol 382:46-76.

Wallen P, Williams TL (1984) Fictive locomotion in the lamprey spinal cord in vitro compared with swimming in the intact and spinal animal. J Physiol (Lond) 347:225-239.

Whelan PJ, Bonnot A, O'Donovan MJ (2000) Properties of rhythmic activity generated by the isolated spinal cord of the neonatal mouse. J Neurophysiol 84:2821-2833.

Yakovenko S, Mushahwar VK, VanderHorst VG, Holstege G, Prochazka A (2002) Spatiotemporal activation of lumbosacral motoneurons in the locomotor step cycle. J Neurophysiol, in press. 